\title{
Numerical investigation on the characteristics of water transfer in PEMFC with bionic flow channel
}

\author{
Tao Chen, Shihua Liu , and $L i$ Yang
}

School of Mechanical and Electronic Engineering, Wuhan University of Technology, Wuhan 430070, China

\begin{abstract}
The content and the transmission characteristics of the water in the polymer electrolyte membrane fuel cell (PEMFC) have a significant influence on its performance. So far, there are few separate numerical studies on the process of water transport in the bionic flow channel, and the performance of bionic flow channel is different from conventional flow channel in part owing to the differences of their water drainage. Therefore, in order to understand the water drainage process of bionic flow channel, two different types of bionic flow channels are adopted in this paper. The method of numerical simulation is used to study the transmission process of liquid water in the bionic flow channel of different placement orientations. The simulation results found that the orientations of bionic flow channel has an influence on the process of water transport in the flow channel, and the process of water drainage also has a significant difference in the two different types of bionic flow channel.
\end{abstract}

\section{Introduction}

The water management has always been the main problem to further enhance the performance of PEMFC[1], in order to deeply understand the influence of the water transfer state and the various control parameters on the water transmission, so as to improve the internal structure and material properties of fuel cell, more and more studies on the water management of fuel cell are emerging, such as the high-tech measurement methods of Xrays, neutron imaging, scanning electron microscopy and nuclear magnetic resonance, etc. Those high-tech measurement methods are adopted to accurately study the transmission process of water in the internal flow channels [2-3], using micro-electro-mechanical systems technology [4-5] and optical fiber sensor [6-8] etc to measure the Water transfer process in the flow channel, more researches are based on visualization technology to observe the state of water transfer in the fuel cell[9-11], although these techniques can be used to study the state of water transfer inside the flow channel, due to the experimental difficulties, and the cost is relatively so higher that these methods are limited to some extent.

Up to date, the method of computer simulation is applied in most studies [12-15], Such as utilizing lattice Boltzmann model[16-18] and volume-of-fluid (VOF) model [19-23] to

Corresponding author: 1shtgyx@163.com 
simulate the process of water transfer in fuel cell, the results of those methods show that the characteristics and phenomena of the water transfer in the flow channel of the fuel cell are both well explained. Anh Dinh Le [19] et al. numerically investigated the process of water transfer in PEMFC with interdigitated flow channel. Theodorakakos A [20] et al. simulated the dynamic process of liquid droplet detachment from porous surface. M. Koz [21] et al. numerically investigated the effects of droplet size in a PEMFC flow field. Cai Y [22-23] et al. numerically investigated the water transfer process in the serpentine flow channel and the straight flow channel. From these studies, on the one hand, through the computer simulation, the detailed process of the liquid water transfer in the flow channel and the factors which affect water transfer can be found. On the other hand, it can be found that the water transfer process of the existing research are focuses on the inner of conventional flow channel, few scholars have carried out the separate simulation study on the water transfer process in the bionic flow channel, and part of the performance of bionic flow channel is different from conventional flow channel owing to the differences of their water drainage [24]. Therefore, the simple numerical study on liquid water in a conventional flow channel is not enough to fully explain the phenomenon of water transport in the bionic flow channel.

With the continuous development of bionic design in recent years, many researchers have applied the bionic design to the flow channel of fuel cell, Ramos-Alvarado et al. [25] applied the constructal theory to the design of flow field. Kloess et al. [26] evaluated the performance of the flow field plate based on the types of lung and leave. Roshandel et al. [27] investigated the performance of the flow field plate based on the type of a leaf. Chen et al. [28] also investigated the performance of leaf type flow field. Guo et al. [29] presented a mathematical model to investigate the performance of leaf type flow field. However, these scholars only conduct the overall test or simulation to the performance of fuel cell which have utilized the method of bionic design, and there is no separate study on the drainage performance in the flow channel of bionic design. From these researches of bionic design, we can not understand enough the water drainage characteristics of flow channel with bionic design, which is not conducive to the design improvement of bionic flow channel and the problem of water management will have a greater effect on the performance of the PEMFC [30].

Therefore, it is significant to investigate the characteristics of water transport in the bionic flow channel. This paper mainly study the transmission process of water in bionic flow channel. Two different types of symmetrical leaf bionic flow channel and asymmetrical leaf bionic flow channel are selected as the research object [31], utilizing the method of numerical simulation to present the transfer process of liquid water in the bionic flow channel, and the influence of different placement orientations of bionic flow channel on water transport has also been studied. All of this can be conducive to a clear understanding of the internal water drainage process of bionic flow channel in detail, it also provides the necessary guidance and basis for the design of bionic flow channel.

\section{Numerical models}

\subsection{VOF model}

The numerical simulations of the 3D, laminar, unsteady, two-phase flow was applied to the computation domain using FLUENT 15.0. The conservation of mass and momentum were applied to the model as the governing equations and the energy equations were not considered. In order to track the two-phase flow interface of gas-water in the domain of computation, the method of volume-of-fluid (VOF) was used [32]. Then, the conservation law of mass and momentum are as follows: 
Continuity equation:

Momentum equation:

$$
\frac{\partial \rho}{\partial t}+\nabla \cdot(\rho \vec{v})=0
$$

$$
\partial(\rho \vec{v}) / \partial t+\nabla \cdot(\rho \vec{v} \vec{v})=-\nabla p+\nabla \cdot(\vec{\tau})+\rho \vec{g}+\vec{F}
$$

where $\vec{F}$ is the momentum source term due to surface tension, $\rho$ is the static pressure and $\vec{\tau}$ is the stress tensor:

$$
\vec{\tau}=\mu\left[\left(\nabla \vec{v}+\nabla \vec{v}^{T}\right)-2 / 3 \nabla \cdot \vec{v} I\right]
$$

Where $I$ is the unit tensor, $\mu$ is the dynamic viscosity .

Volume fraction of water $\alpha_{2}$ can be solved as follows:

$$
\frac{\partial \alpha_{2}}{\partial t}+\vec{v} \cdot \nabla \alpha_{2}=0
$$

The volume fraction of gas $\alpha_{1}$ can be solved by following equation:

$$
\alpha_{1}+\alpha_{2}=1
$$

The surface tension of phase and wall has an important influence on the transmission process of two-phase flow which is included in the VOF model[32]. In the paper, the model of continuum surface force which is proposed by Brackbill et al. [33] was used.

\subsection{Geometric model}

Figure 1 is a model of two different bionic flow channel structures. These two bionic structures are derived from Ref. [31]. A water inlet hole of $0.2 \mathrm{~mm}$ diameter is arranged on the wall near the inlet of the bionic flow channel (The wall is the interface between the channel and the gas diffusion layer (GDL) ) [23]. FLUENT 15.0 is used to simulate water dynamic in the bionic flow channels. The number of meshes of symmetrical leaf bionic flow channels is 221674 , and The number of meshes of asymmetrical leaf bionic flow channels is 232583 . Accuracy of the model tested by increasing and reducing the number of meshes by $20 \%$, and the similar processes of water transport were obtained.

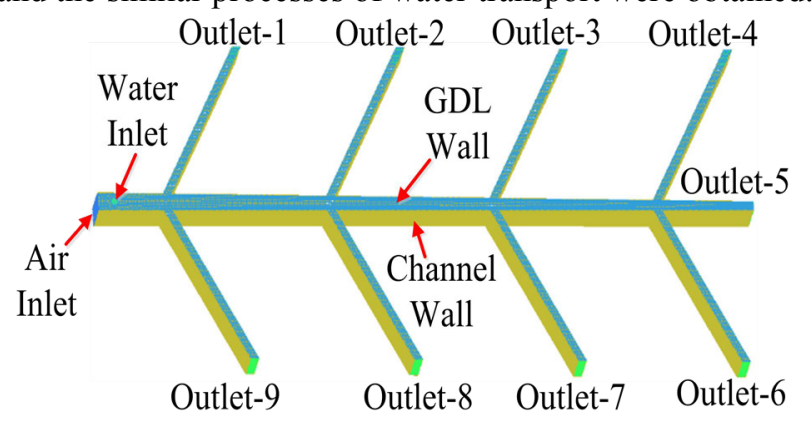

(a) Symmetrical leaf bionic flow channel 


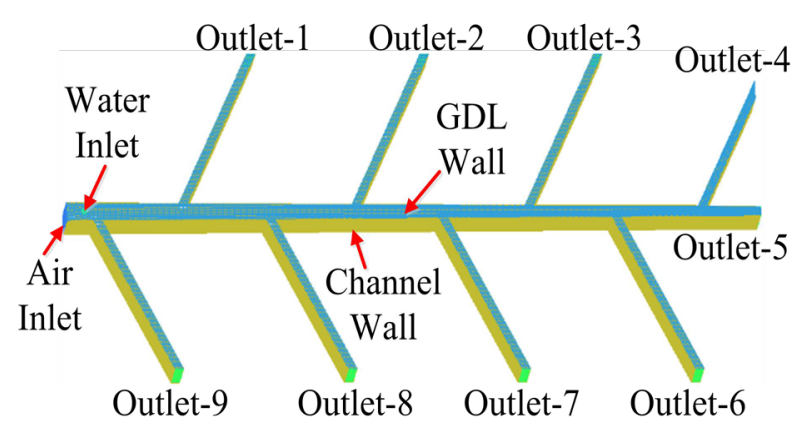

(b) Asymmetrical leaf bionic flow channel

Fig. 1. Schematic of two different bionic flow channel

\subsection{Geometric model}

In the parameter settings of the simulation, a gas velocity of $10 \mathrm{~ms}^{-1}$ normal to the boundary is used[34]. Moreover, according to the theoretical calculation of the velocity of water to be about $1.8 \times 10^{-6} \mathrm{~ms}^{-1}$, which is too small compared to $10 \mathrm{~ms}^{-1}$ of gas inlet [35]. To accelerate the speed of computational, the velocity of water with $0.1 \mathrm{~ms}^{-1}$ normal to the boundary is used in this study. The gauge pressure at the outlet of the flow channel is set to $0 \mathrm{~Pa}$. In all the computational cases, the gravity is considered all the time according to the different locations of the flow channel placement. The contact angle of the surface of GDL and flow channel wall were $140^{\circ}$ and $45^{\circ}$, respectively [36]. The time step was set at $10^{-6} \mathrm{~s}$ in order to guarantee that the global courant number is less than one. The surface tension at the gas-liquid interface is $0.072 \mathrm{Nm}^{-1}$. In order to solve the governing equations, the pressure velocity coupling method via the PISO scheme is used.

\section{Results and discussion}

The characteristics of water flow in the bionic flow channel are simulated. The five cases corresponding to different orientations of bionic flow channel placement were simulated, as listed in Table.1. Detailed results and discussions are as follows.

Table 1. The gravity in different orientations of leaf bionic flow channel placement

\begin{tabular}{c|c|c|c}
\hline & $\begin{array}{c}\mathbf{X} \text { axial } \\
\left.\text { gravity/(ms } \mathbf{2}^{-2}\right)\end{array}$ & $\begin{array}{c}\mathbf{Y} \text { axial } \\
\text { gravity } /\left(\mathbf{m s}^{-2}\right)\end{array}$ & $\begin{array}{c}\mathbf{Z} \text { axial } \\
\text { gravity } /\left(\mathbf{m s}^{-2}\right)\end{array}$ \\
\hline Side & 0 & -9.8 & 0 \\
Inversion & -9.8 & 0 & 0 \\
Reversal & 0 & 0 & 9.8 \\
Perpendicularity & 9.8 & 0 & 0 \\
Level & 0 & 0 & -9.8 \\
\hline
\end{tabular}

\subsection{The state of water in the symmetrical leaf bionic flow channel in different orientations and different time}

Fig. 2 is the state of the water in the symmetrical leaf bionic flow channel in five different orientations (side, inversion, reversal, perpendicularity and level) and different 
time. From Fig. 2-a, at about $3 \mathrm{~ms}$, the droplets in a state of constant expansion hardly move. Since the wall of the water inlet is the surface of the GDL, the volume of the droplets at the beginning is very small, and the force of gas flow exerting on the droplet is not enough to get rid of the adsorption force between the droplet and the surface of the GDL. Only when the liquid droplet grows up to a certain volume before the apparent movement, and this situation also occurred in the Fig. 2-b, c, d, e. From Fig. 2-a, b, c, d, e (symmetrical leaf bionic flow channel in the state of side, inversion, reversal, perpendicularity and level respectively) can be seen, at about $9 \mathrm{~ms}$, the droplets move mostly in the branch flow channels ( outlet-1 and outlet-9) where near the gas inlet, it can be also seen from Fig. 11 that the pressure difference in the branch flow channel nearer the gas inlet is larger, and it may also explain the reason why the droplets move faster in the branch flow channel where nearer the gas inlet.

However, from Fig.3, before the $11 \mathrm{~ms}$ time, no matter what kind of orientations of the symmetrical leaf bionic flow channel, their maximum velocity curves are almost identical. After $11 \mathrm{~ms}$, the maximum velocity of the internal flow under the condition of different flow channel placement has a fluctuation. At about $15 \mathrm{~ms}$, from Fig. 2, there are water droplets in each branch flow channel (such as outlet-1 and outlet-9 etc.) where near the gas inlet of the symmetrical leaf bionic flow channel. There are few droplets in the branch flow channel (such as outlet-4 and outlet-6 etc.) where is far away from the gas inlet. It shows that the water in the symmetrical bionic flow channel is mainly discharged from the branch flow channel where near the gas inlet. What's more, it can be also seen from Fig. 11 that the pressure difference is very small in the branch flow channel where is far away from the gas inlet, and we can infer that the velocity of the liquid water flow in these flow channels is the slowest, Once liquid water accumulates in the branch flow channel where is far away from the gas inlet is difficult to be discharged rapidly, so that the water content is higher in these branch flow channels. It is the same to the results obtained by other literature that there are higher water content in the branch flow channel where is far away from the gas inlet $[24,26,37]$.

In addition, from Fig.4, it shows the volume of water in the symmetrical flow channel in the different orientations and different time, and when the flow channel is in the orientation of perpendicularity, the performance of water drainage is the best, because the direction of the droplet gravity is consistent with the direction of gas flow. So, when designing symmetrical bionic flow channel which branch flow channel should be closer to the gas inlet and the number of branches along the main flow channel shouldn' t be too many. Only in this way, it is possible to avoid the pressure of the branch flow channel which is far away from the gas inlet too small, so as to keep the flow channel well drained. 

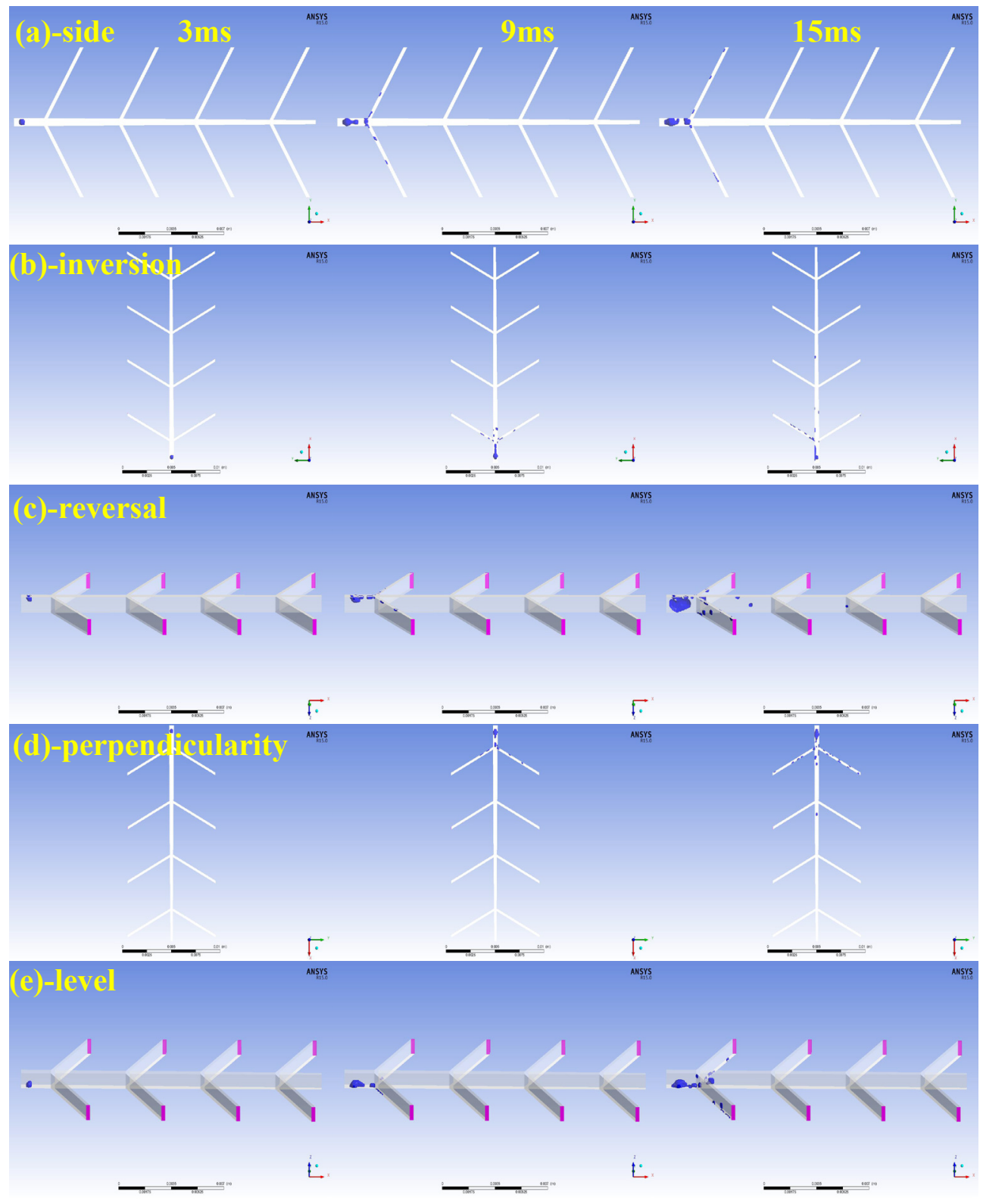

Fig. 2. The state of water in the symmetrical flow channel in different orientations and different time 


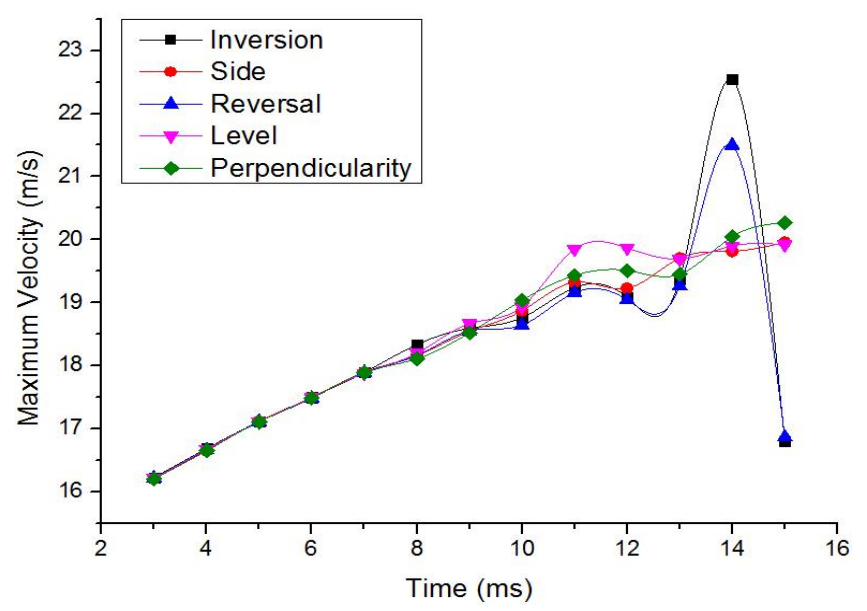

Fig. 3. The maximum velocity in the symmetrical flow channel in the different orientations and different time

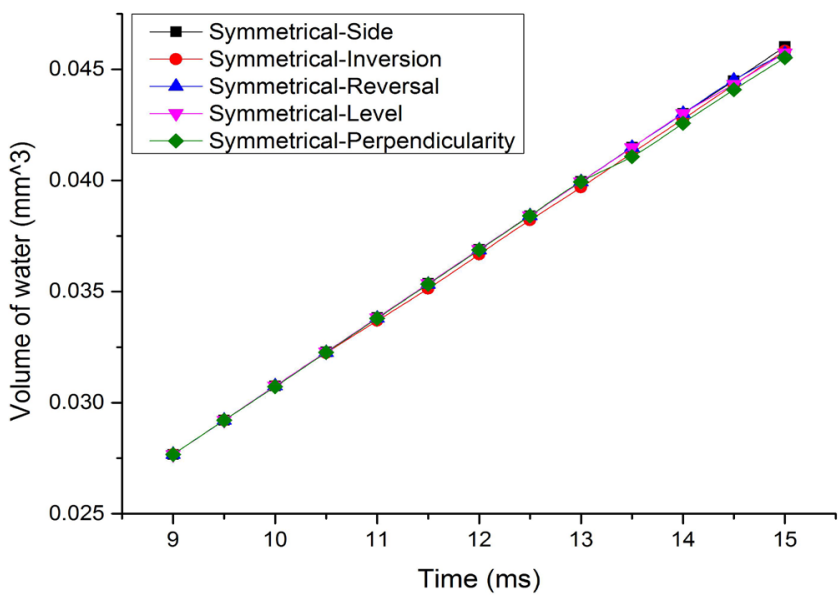

Fig.4. Volume of water in the symmetrical flow channel in the different orientations and different time

\subsection{The state of water in the asymmetrical leaf bionic flow channel in different orientations and different time}

Fig. 5 is the state of water at different time in five different orientations (side, inversion, reversal, perpendicularity and level) in an asymmetrical leaf bionic flow channel. As the same shown in Fig. 2, the droplets barely move at about $3 \mathrm{~ms}$. At about $9 \mathrm{~ms}$, there are significant differences in the distribution of liquid water droplets between Fig. 4-a, c, d (the asymmetrical leaf bionic flow channel in side, reversal and perpendicularity orientations ) and 4-b, e (the asymmetrical leaf bionic flow channel in inversion and level orientations), and it can be seen that the position of liquid water droplets is almost consistent with the time of $3 \mathrm{~ms}$ in Fig.5-b, e. That is to say that the liquid water moves slowly when the flow channel was placed in the state of inversion and Level. As shown in Fig.5-a, c, d, the liquid water mainly distribute in the main flow channel of bionic flow channel and the velocity is faster. 


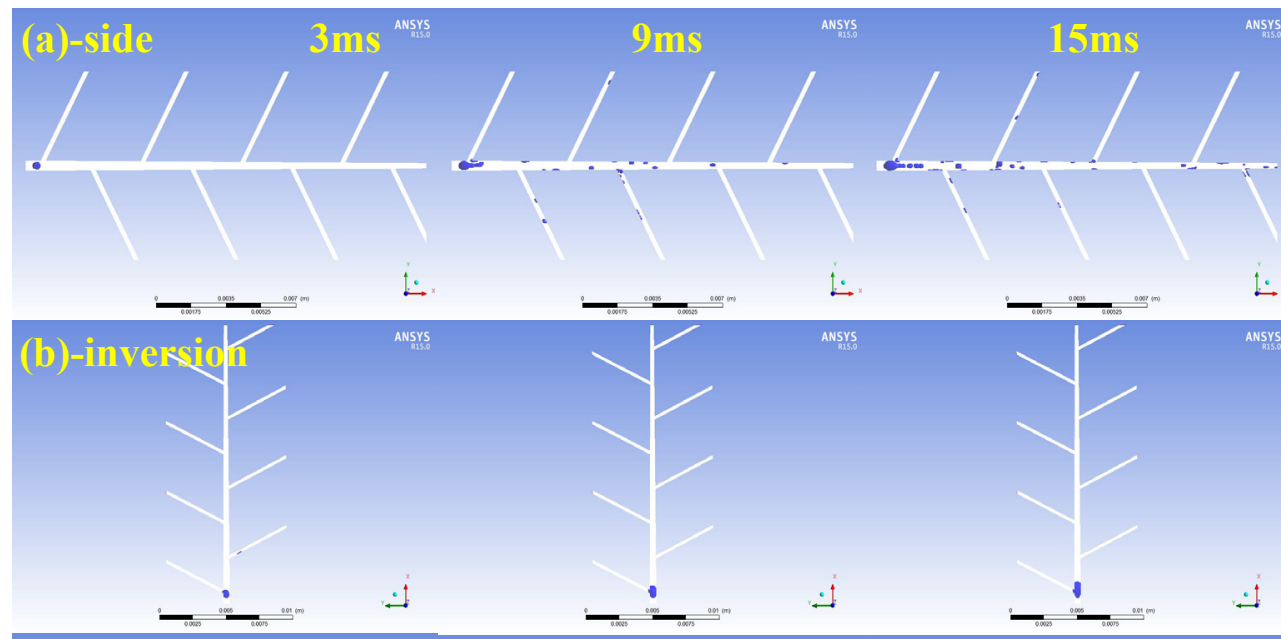

\section{(c)-reversal}
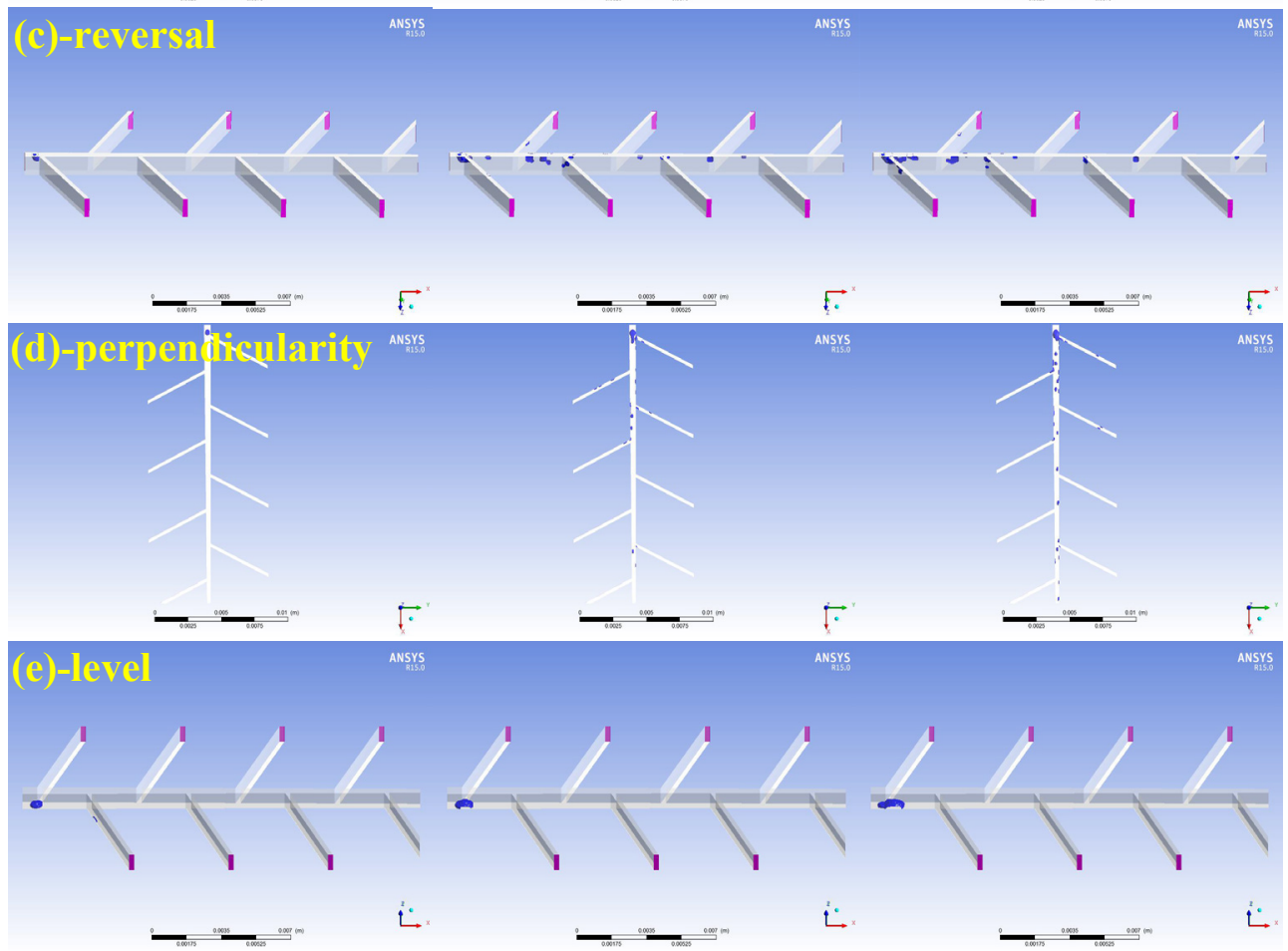

Fig.5. The state of water in the asymmetrical flow channel in different orientations and different time

It can be seen from Fig. 6 that the maximum velocity inside the flow channel which are the positions of side, reversal and perpendicularity is significantly faster than that in inversion and level positions. Because of the different orientations of flow channel, the directions of gas blowing force and gravity on the liquid water are different. It can be drawn that the gravity has a large effect on the movement and distribution of water droplets inside the channel when the asymmetrical leaf bionic flow channel is in different orientations. At about $15 \mathrm{~ms}$, it can be seen that the distance to gas inlet count for little the distribution of liquid water in branch flow channel, which is greatly different to the phenomenon that the 
shorter of the distance to gas inlet, the more liquid water in branch flow channel of symmetrical leaf bionic flow channel. As shown in Fig.5-b, e, there are little difference in the movement of liquid water between inversion and level orientations in asymmetrical leaf bionic flow channel at about $15 \mathrm{~ms}$. In addition, it can be concluded from the Fig. 12 that pressure difference in each branch flow channel reduce along the direction of main flow channel in asymmetrical leaf bionic flow channel. Once liquid water accumulates in the branch flow channel which is far away from the gas inlet is difficult to be discharged rapidly, Therefore, the water in these branch flow channels is higher, and it can be drawn the same conclusions from other literature [24,26,37]. In addition, from Fig.7, it shows the water volume in the symmetrical flow channel in the different orientations and different time, and when the flow channel is in the orientation of perpendicularity, the performance of water drainage is the best, because the direction of the droplet gravity is consistent with the direction of gas flow. In conclusion, to avoid the pressure difference too low to drain liquid water from branch flow channel, the number of branches along the main flow channel shouldn't be too many in design of asymmetrical bionic flow channel.

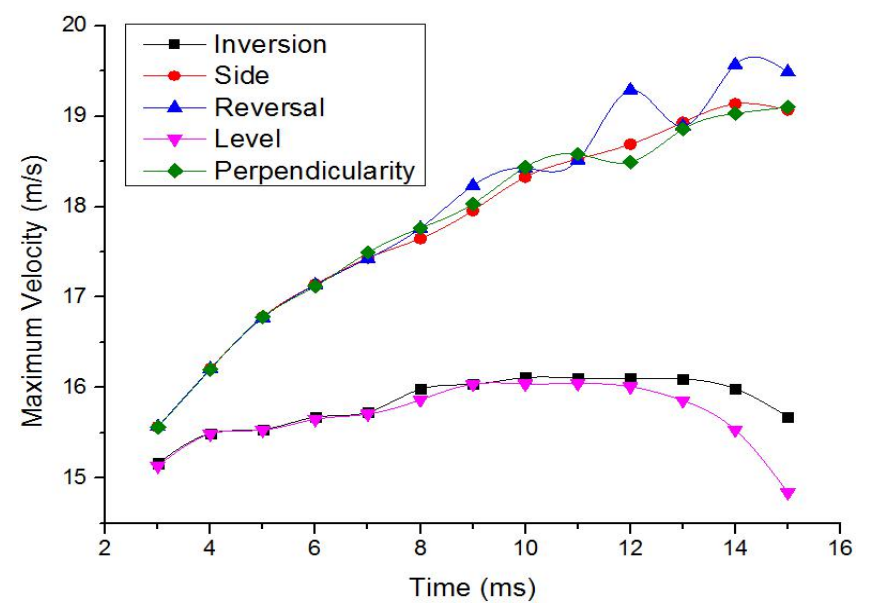

Fig.6. The maximum velocity in the asymmetrical flow channel in the different orientations and different time

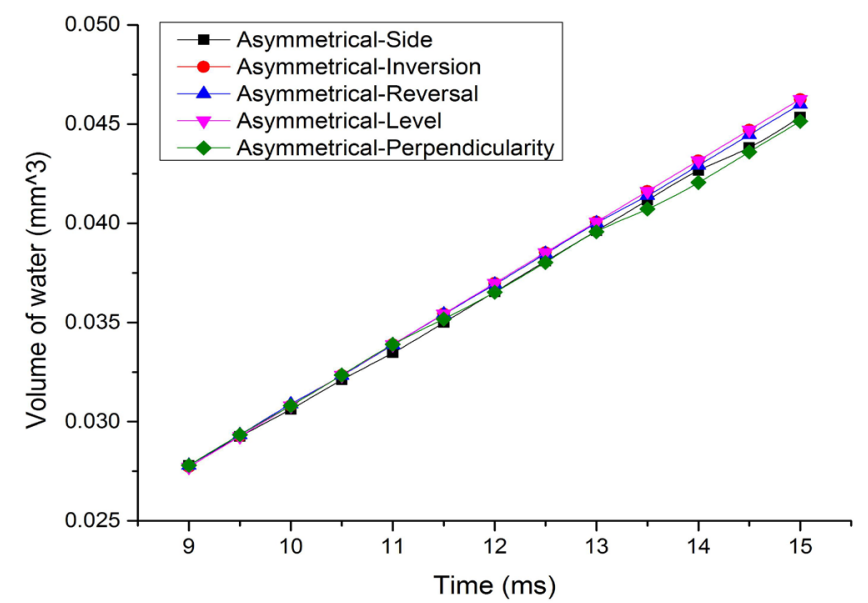

Fig.7. Volume of water in the asymmetrical flow channel in the different orientations and different time 


\subsection{The state of water in the flow channel of symmetrical and asymmetrical structures in the same orientation}

In order to compare the state of water droplets at different time and same orientations more clearly in the bionic flow channel for symmetrical and asymmetrical of leaf type, followed a detailed comparison of the state of water movement in the two different bionic flow channels at different time. From Fig.8-a, Fig.8-c and Fig. 8-d, it can be seen that when the two bionic flow channels are in the three kinds of situations of side, reversal and perpendicularity respectively, the water inside the channel moves faster. However, the obvious difference of water distribution between the two channels can be seen in the three situations. For the water in the flow channel of symmetrical leaf bionic is mainly distributed in the branch flow channel where near the gas inlet, and there is almost no liquid water in the branch flow channel which is far away from the gas inlet, that is to say, comparing with the branch flow channel where far away from the gas inlet, most of the liquid water were drained from the branch flow channel where near the gas inlet. It also shows that the distance between gas inlet and branch flow channel has a great influence on the water distribution in the symmetrical leaf bionic flow channel. The liquid water in the asymmetrical bionic flow channel is mainly distributed in the main flow channel, and the other branch flow channel also has the presence of liquid water, in addition, the distribution of liquid water in the branch flow channel is no significant influence by the distance between the branch flow channel and the gas inlet, there is significant difference when compares with the situation that water is mainly distribution in the branch flow channel where near the gas inlet for the symmetrical leaf bionic flow channel, it can be seen clearly that the difference of water distribution between the two kinds of flow channels at about $15 \mathrm{~ms}$. As it can be seen in Fig.8-b and Fig.8-e, when the two types of bionic flow channel were placed in inversion and level situation that also appeared obvious differences between the movement and the distribution of liquid water. The water were drained mainly from the branch flow channel where near the gas inlet for symmetrical leaf bionic flow channel in the situations of inversion and level placement, comparing to the case of the same placement of the asymmetrical bionic flow channel, the velocity of liquid water is faster, and the velocity of the liquid water movement in the asymmetrical bionic flow channel of the inversion and the level position is the slowest.

It also can draw from Fig.9, no matter what positions for the two kinds of bionic flow channels, the maximum velocity of the water in the symmetrical leaf bionic flow channel is almost higher than that in the asymmetrical leaf bionic flow channel. Especially, form Fig.10, it shows that the performance of water drainage is the best for the position of perpendicularity in asymmetrical flow channel, And it can be seen from Fig.13, the pressure in the main flow channel of the symmetrical leaf bionic flow channel is in a state of fluctuation, and the amplitude of the fluctuation is larger and nearly identical for each placement of the flow channel. However, the pressure in the asymmetrical bionic flow channel is in a relatively stable state, and the pressure fluctuation, although there is a slight difference in the pressure of each channel. Therefore, the difference of pressure fluctuation and steady change in the main flow channel of the two types of bionic flow channel may be one of the cases which cause the difference of water distribution finally. In other words, the fluctuation of pressure has some influence on the process of water drainage inside the flow channel, the evidence can also be obtained from other studies[38]. 


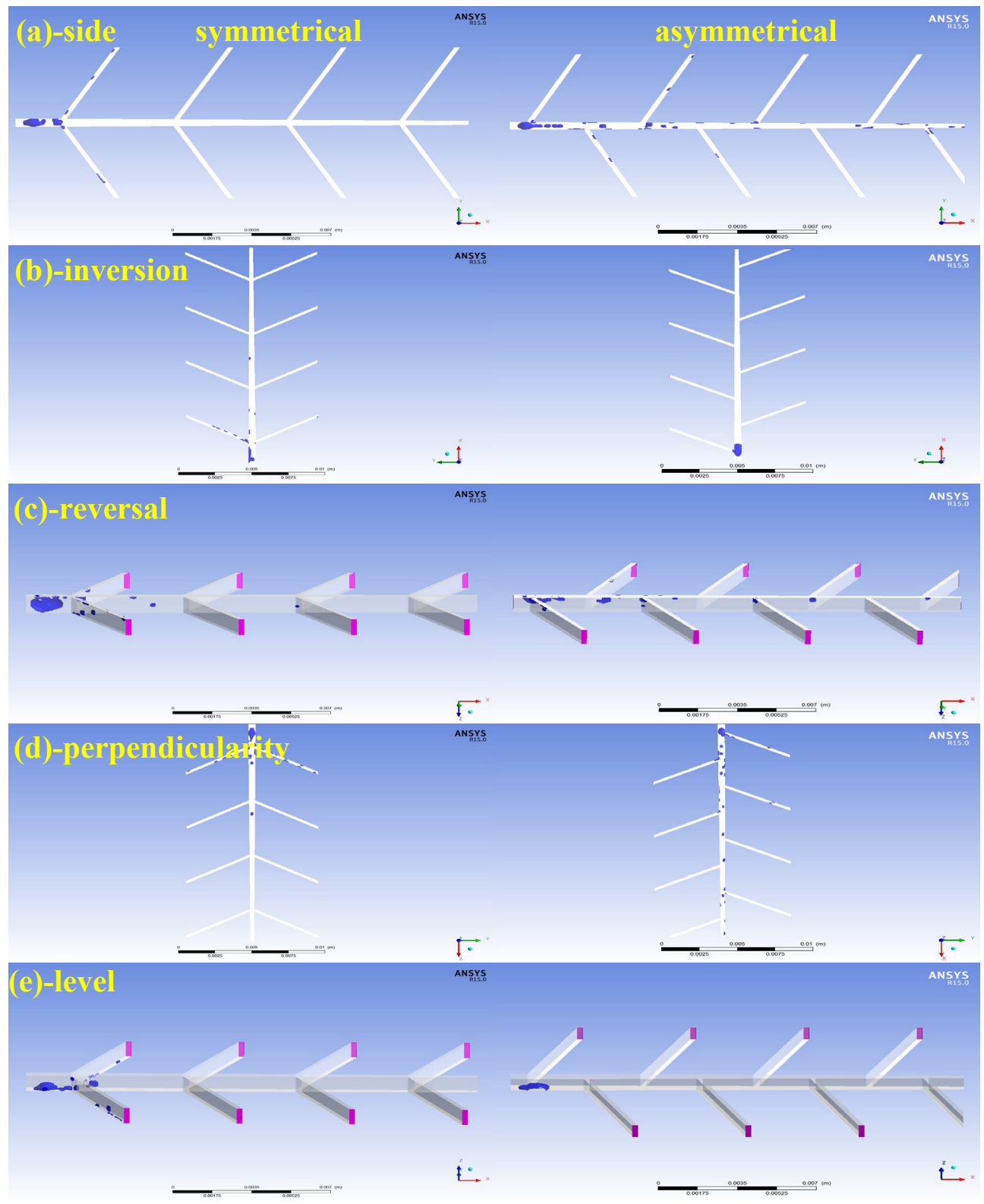

Fig.8. The state of water in flow channel of symmetrical and asymmetrical structures in different orientations and the time of $15 \mathrm{~ms}$ 


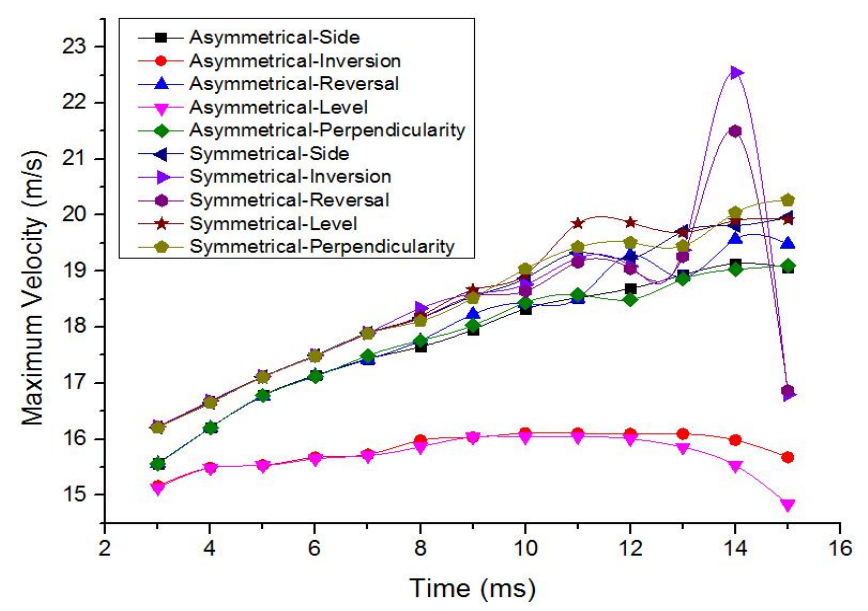

Fig.9. The maximum velocity in flow channel of symmetrical and asymmetrical structures in different orientations

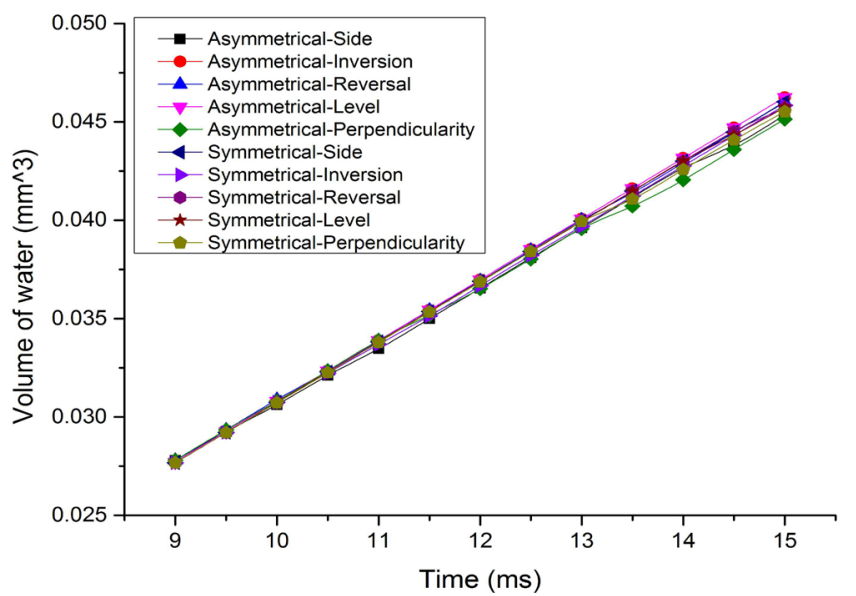

Fig.10. Volume of water in flow channel of symmetrical and asymmetrical structure in different orientations and different time 


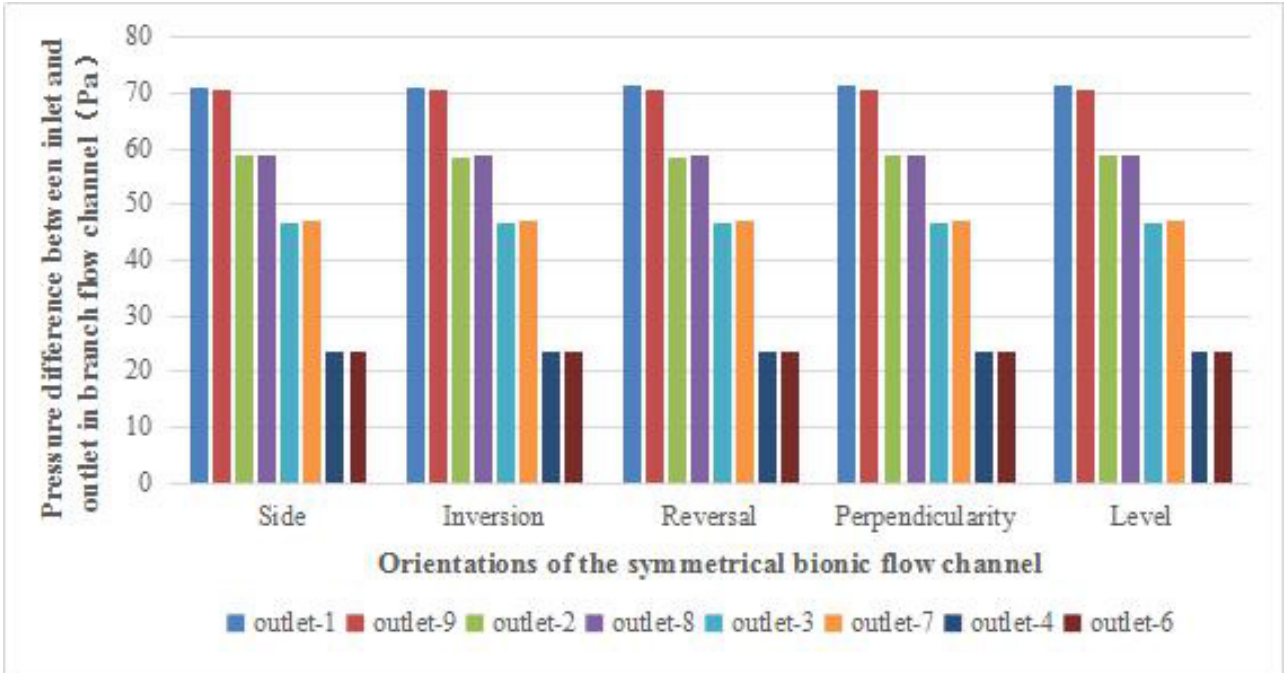

Fig. 11. The pressure difference in each branch flow channel of symmetrical structure in the different orientations

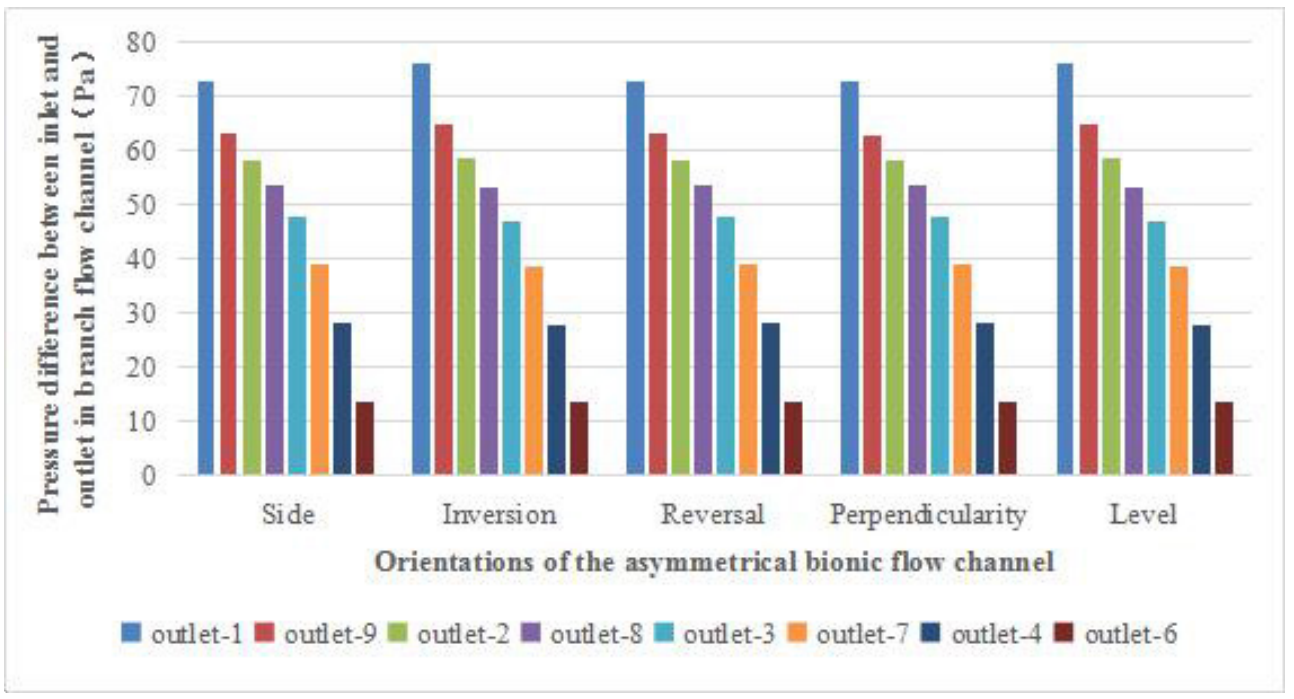

Fig.12. The pressure difference in each branch flow channel of asymmetrical structure in the different orientations 


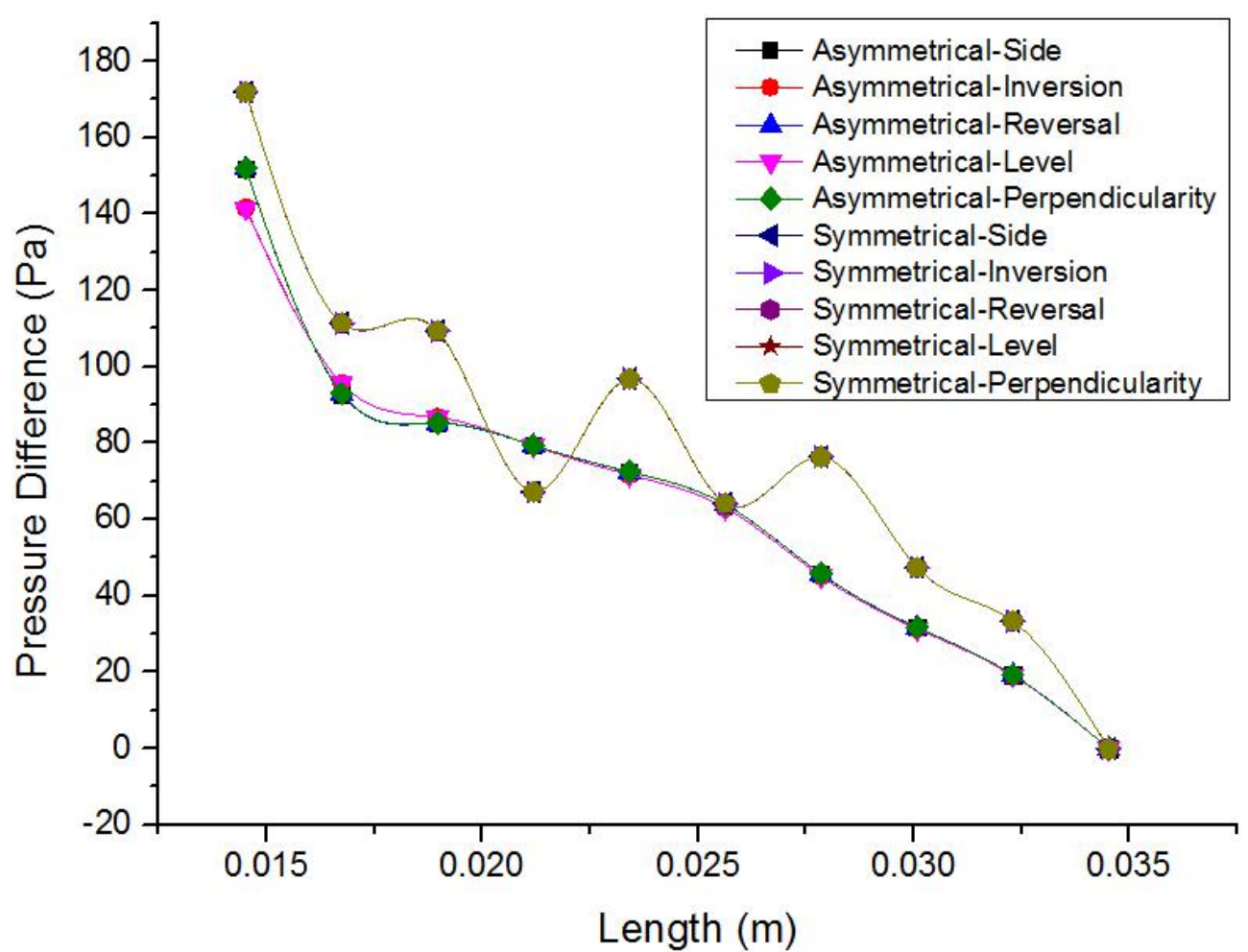

Fig. 13. The pressure difference in each branch flow channel of asymmetrical structure in the different orientations

\section{Conclusion}

This paper is mainly focused on the comparison and analysis of the flow and distribution of liquid water in the symmetrical and the asymmetrical of leaf bionic flow channel in the different orientations(side, inversion, reversal, perpendicularity and level). The conclusions are as follows:

(1) By comparing and analyzing the distribution and movement of the liquid water in leaf bionic flow channel of the symmetrical and the asymmetrical structures, it is concluded that there are significant differences. For the type of symmetrical leaf bionic flow channel, comparing with the branch flow channel where far away from the gas inlet, water is mainly distributed in the branch flow channel which near the gas inlet, and there is almost no liquid water in the branch flow channel which is far away from the gas inlet.

(2) The water in the asymmetrical bionic flow channel is mainly distributed in the main flow channel, the other branch flow channel also has the presence of liquid water. In addition, the distribution of liquid water in the branch flow channel has no significant influence by the distance between the branch flow channel and the gas inlet. and when the two types of bionic flow channel are placed in the positions of inversion and level respectively, the maximum velocity in the internal symmetrical bionic flow channel is far greater than that in the asymmetrical bionic flow channel.

(3) As shown in Fig.13, the pressure difference in the two different types of flow channel presents a state of fluctuation and slow change, the difference of pressure fluctuation and steady change in the main flow channel of the two types of bionic flow channel may be one of the cases which cause the difference of water distribution finally. In 
other words, the fluctuation of pressure has some influence on the process of water drainage inside the flow channel, the evidence can also be obtained from other studies [38].

(4) It can also be known by the pressure analysis that the pressure difference in the main flow channel of symmetrical and asymmetrical is more smaller when the distance is more larger between the branch flow channel and gas inlet. In conclusion, to avoid the pressure drop too low to drain liquid water easily, the number of branches along the main flow channel shouldn't be too many in design of symmetrical and asymmetrical leaf bionic flow channel. Moreover, no matter what kinds of orientations of the symmetrical and asymmetrical leaf bionic flow channel, the liquid water in the bionic flow channel is easier to be drained when the flow channel is in the position of perpendicularity.

Totally, the results of the present work would be beneficial in design of bionic flow channel for PEMFC.

\section{Acknowledgments}

The authors would like to thank the National Science Foundation of China [No.51575413] and the Wuhan Science and Technology Bureau in China [No.2018010401011329] for funding this study.

\section{References}

1. K Jiao, X Li, PROG ENERG COMBUST. 37, 221-291 (2011)

2. I Manke, C Hartnig, N Kardjilov, Fuel Cells. 10, 26-34 (2010)

3. M Saito, A Kikuko Hayamizu, T Okada, J PHYS CHEM B. 108, 16064-16070 (2005)

4. $\mathrm{CH}$ Chen , CY Lee, S Lee, YM Liu, INT J HYDROGEN ENERG. 39, 18337-18342 (2014)

5. Lee C, Lee Y, INT J HYDROGEN ENERG. 37, 4448-4456 (2012)

6. K Inman, X Wang, INT J HYDROGEN ENERG. 39, 19691-19700 (2014)

7. N David, KV Schilling, PM Wild, N Djilali, INT J HYDROGEN ENERG. 39, 1763817644 (2014)

8. N David, N Djilali, MICROFLUID NANOFLUID. 13, 99-106 (2012)

9. K Nishida, T Murakami, S Tsushima, S Hirai, Electrochemistry. 75, 149-151 (2007)

10. H Masuda, K Ito, T Oshima, K Sasaki, J POWER SOURCES. 177, 303-313 (2008)

11. S Lee, DH Rhee, WH Pyen, HH Cho, J MECH SCI TECHNOL. 25, 957-961 (2011)

12. T Chen, S Liu, S Gong, C Wu, INT J ENERG RES. 37, 1680-1688(2013)

13. A Su, YC Chiu, FB Weng, INT J ENERG RES. 29, 409-425 (2010)

14. YM Ferng, A Su, SM Lu, INT J ENERG RES. 32, 12-23 (2010)

15. WJ Yang, SJ Kang, YB Kim, INT J ENERG RES. 36, 1051-1064 (2012)

16. HB Han, M Ni, H Meng, Entropy. 18, 17(2016)

17. Song Bao-Wei, Ren Feng, Hu Hai-Bao, Qiao-Gao H, J POWER SOURCES. 278, 703717 (2015)

18. B Han, H Meng, INT J HYDROGEN ENERG. 38, 5053-5059 (2013)

19. AD Le, B Zhou, J POWER SOURCES. 193, 665-683 (2009)

20. A Theodorakakos, T Ous, M Gavaises, J COLLOID INTERF SCI. 300, 673-687 (2006)

21. KG Maler, Ecs Transactions. 50, 183-196 (2013) 
22. JY Cai, T Chen, T Yang, J Xiao, J POWER SOURCES. 209, 90-104 (2012)

23. Y Cai, T Yang, PC Sui, J Xiao, INT J HYDROGEN ENERG. 41, 16220-16229 (2016)

24. H Ruan, C Wu, S Liu, T Chen, HEAT MASS TRANSFER. 52, 1-10 (2016)

25. B Ramos-Alvarado, A Hernandez-Guerrero, F Elizalde-Blancas, INT J HYDROGEN ENERG. 36, 12965-12976 (2011)

26. JP Kloess, X Wang, J Liu, Z Shi, L Guessous, J POWER SOURCES. 188, 132-140 (2009)

27. R Roshandel, F Arbabi, G K Moghaddam, RENEW ENERG. 41, 86-95 (2012)

28. T Chen, Y Xiao, T Chen, Energy Procedia. 28, 134-139 (2012)

29. N Guo, MC Leu, M Wu. 607-623 (2011)

30. HS Kim, K Min, J MECH SCI TECHNOL 28, 3837-3843 (2014)

31. XQ Wang, P Xu, AS Mujumdar, C Yap, INT J THERM SCI. 49, 272-280 (2010)

32. Fluent 6.1 User's Guide, Fluent Inc.

33. J U Brackbill, D B Kothe, C Zemach, J COMPUT PHYS. 100, 335-354 (1992)

34. X Zhu, P C Sui, N Djilali, MICROFLUID NANOFLUID. 4, 543-555 (2008)

35. K Jiao, B Zhou, J FUEL CELL SCI TECH. 5, 41011 (2008)

36. B Sundén, M Faghri, Energy Resources (Apr). 175-213 (2005)

37. N Guo, M C Leu, U O Koylu, INT J HYDROGEN ENERG. 39, 21185-21195 (2014)

38. J W Choi, Y S Hwang, S W Cha, S K Min, Fuel \& Energy Abstracts. 35, 12469-12479 (2010) 